\title{
Multiculturalismos, procesos de comunicación intercultural y derechos humanos en los itinerarios de atención en salud del pueblo Iny que vive en el municipio de Aruanã
}

\section{Jacqueline Isabel Ledesma Correa}

\section{RESUMEN}

Entre los años 2012 y 2014 realicé una etnografía para conocer las estrategias de resolución de los procesos de salud y enfermedad del pueblo Iny-Karajá que vive en el Municipio de Aruanã situado en el Estado de Goiás en la región Centro Oeste de Brasil. Este artículo intenta aproximarse a una descripción sobre los diversos "multiculturalismos" y modalidades de comunicación intercultural que se presentan en los servicios de salud pertenecientes al Distrito de Saúde Araguaia. Considerando que el campo de la salud indígena es transversalizado por los Derechos Humanos se plantean algunas reflexiones vinculando ambos campos de problemas.

PALABRAS CLAVE: Salud indígena. Procesos de comunicación intercultural. Derechos humanos.

\section{Multiculturalismos, processos de comunicação intercultural y direitos humanos nos itinerários de atenção à saúde do povo Iny que vive no município de Aruanã}

RESUMO

Entre 2012 e 2014, realizei uma etnografia para investigar as estratégias de resolução dos processos de saúde e doença do povo Iny-Karajá que mora no município de Aruanã, localizado no estado de Goiás, na região Centro-Oeste do Brasil. Este artigo busca descrever os vários "multiculturalismos" e modalidades de comunicação intercultural que são apresentados nos serviços de saúde pertencentes ao Distrito Saúde Araguaia. Considerando que o campo da saúde indígena é predominante pelos direitos humanos, são levantadas algumas reflexões que ligam os dois campos de problemas.

PALAVRAS-CHAVE: Saúde indígena. Processos de comunicação intercultural. Direitos humanos. 


\section{Contextualización}

El proyecto de investigación que sustenta este artículo se desarrolló durante mi proceso de pos-graduación en Antropología Social en la (OMITIDO PARA FINS DE AVALIAÇÃO A CEGAS).

El estudio se enfocó en la experiencia de relacionamiento del pueblo Iny con los recursos que ofrecen las políticas públicas de Salud Indígena ejecutadas por el Sistema de Atenção em Saúde Indígena (SASI). Teniendo en cuenta en relación a este eje las concepciones de salud y enfermedad que los interlocutores de la comunidad Iny de Aruanã, expresaron mediante narrativas orales y visuales.

Iny es la forma lingüística en que se presentan muchos integrantes del pueblo originario del Río Araguaia, significa: "nosotros, gente verdadera”. Karajá es un etnônimo tupi-guaraní cuya traducción es: "macaco grande". El trabajo de campo convoco a un grupo de familias Iny y los agentes de salud que trabajan en el SASI, indígenas y no indígenas. Los escenarios de campo comprendieron: las Aldeas Buridina y Bdè-Brè; un puesto de Salud Indígena, el Hospital Municipal y el Centro de Salud ubicados en la ciudad de Aruanã; además de la Casa de Saúde do Indio (CASAI) situada en la ciudad de Goiânia.

Visité la Escola Maurehi ubicada en el predio de la Aldea Buridina en la cual por la mañana los alumnos reciben sus clases en Inybire (lengua Iny). Fui invitada por dos maestras de artesanato a las aulas de confección de objetos en cerámica y trenzado en palha (fibra vegetal) que ellas dirigen. Iraci esposa del cacique Raúl de la aldea Buridina coordina la enseñanza de tejido en palha para adultos, jóvenes y niñas, en un espacio techado al aire libre ubicado cerca de la Escola Maurehi. Kari Wassuri que vive en la aldea Bdè-Brè enseña cerámica sobre una mesa con caballetes al frente de la casa de su hermano Renán situada en la aldea Buridina frente al río Araguaia.

Ambas maestras consideran que la confección y aprendizaje del tejido en palha y cerámica constituyen prácticas saludables y "terapéuticas" para ellas y el entorno de las aldeas. Vinculan estas actividades con la salud, cuidado y trasmisión de la tradición del Pueblo Iny y destacan su importancia como fuente de ingresos, mediante la venta de la producción de piezas de artesanato. 
La relación del Pueblo Iny con el mundo Tori, término con el cual nombran a las personas no indígenas en particular a: "o branco”, está señalado históricamente por procesos de colonización violenta, física, simbólica y cultural. Los Iny han luchado y resistido como otros pueblos originarios de las Américas reclamando sus territorios, reivindicando su autonomía, protegiendo su identidad lingüística y tradiciones culturales.

La Constitución de la República Federativa de Brasil promulgada el 5 de octubre de 1988 reconoció el derecho de los pueblos indígenas a sus tierras, políticas sociales adecuadas a sus especificidades culturales, auto- representación jurídico-política y preservación de su identidad cultural (GARNELO et al., 2012). Fue la primer Carta Magna que otorga un capítulo específico, a la protección de los derechos indígenas, reconociendo la capacidad de autodeterminación y organización de los Pueblos Indígenas para defender sus intereses, asignando al Ministerio Público el deber de garantirlos (TORRES et.al.2008).

Al final de la década de 1980 varios países de la región proclaman nuevas constituciones y/o reforman las existentes, dando lugar a la "cuarta ola de constituciones" y al fenómeno calificado por la politóloga norteamericana Donna Lee Van Cott de: “constitucionalismo multicultural” (CEPAL, 2017).

En el año 1999 Brasil efectúa un avance muy importante en relación al derecho a la salud de los pueblos indígenas, formula la política nacional de Salud Indígena reglamentada con la Ley Arouca número 9.836, declarando garantizar el respeto a exigencias particulares sobre los procesos de salud y enfermedad indígena en sus contextos específicos (BARROS, 2011).Esta ley crea el SASI, formaliza la creación de los Distritos Sanitários Especiáis Indigenas (DSEI) instituye la representación indígena en los Conselhos Estaduais de Saúde (CES) y Conselhos Municipal de Saúde (CMS) (ibíd.).

La forma de organización del modelo de atención en salud indígena se nutre de proyectos con inspiración del Sistema Único de Saúde (SUS), la participación de la Fundação Nacional do Índio (FUNAI), ONGS, Universidades y organizaciones indigenistas (VERANI, 1999).

\section{Políticas en salud indígena, multiculturalismos y comunicación intercultural}

\footnotetext{
O estado de saúde e doença para os povos indígenas, em seu principal aspecto, é o resultado do tipo de relação individual e coletiva que se estabelece com as demais pessoas e com a natureza (Baniwa, 2006).
}

Dossiê Redes de Inter-aprendizaje: nuevas cartografías interculturales y algunas propuestas de transformación R. Articul.const.saber, 2020, v.5: e65684 
Los Iny con los que se construyó esta pesquisa diseñaron la salud y la enfermedad $\mathrm{y}$ en algunas ocasiones solicitaron ser fotografiados. Las narrativas visuales y orales conforman mensajes estéticos, éticos y políticos en las que manifiestan sus experiencias en los itinerarios interculturales en salud/enfermedad y educación.

Figura 1 - Aula de cerámica dirigida por Kari Wassuri, referente comunitaria Iny. Armando onzas y personajes de la cosmología Iny.

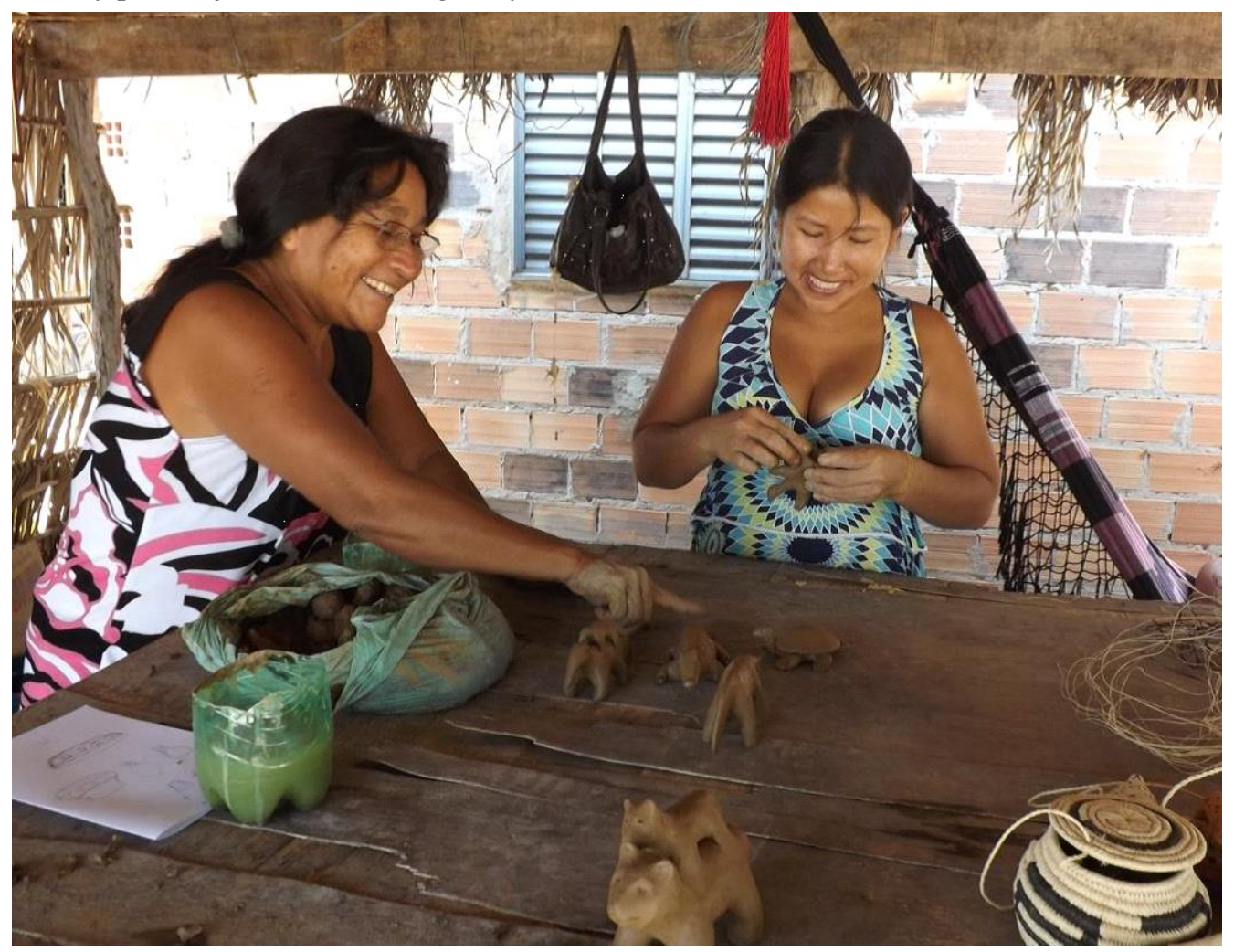

Fuente: Jacqueline Ledesma (Octubre 2013).

Al momento del trabajo etnográfico los líderes Iny de las aldeas de Aruanã, consideran que los proyectos de salud y educación siempre tienen tutores y los nombran: FUNAI, Ministério da Educação (MEC), FUNASA, Municipios. “Tudos nos tutelam”.

El campo de la Salud Indígena en Brasil ha convocado a lo largo de su historia la intervención de múltiples entidades: estatales, religiosas, civiles; los pueblos indígenas surgen como "Protagonistas" en las normativas, demandas y decisiones luego de un prolongado "camino de fuerte tutelaje".

Dossiê Redes de Inter-aprendizaje: nuevas cartografías interculturales y algunas propuestas de transformación R. Articul.const.saber, 2020, v.5: e65684 
El Convenio sobre Pueblos Indígenas y Tribales núm. 169 de la OIT del año 1989 (ratificado en Brasil en el año 2004) plantea en sus artículos 6, 7 y 15, que los Estados deben fortalecer los mecanismos de consulta necesarios a los Pueblos Indígenas en lo relativo a sus tierras y recursos naturales, además de favorecer formas para que participen libremente en las decisiones de instituciones y los diseños de programas y políticas que los afecten y sean de su interés. El Consejo de Administración de la OIT ha declarado que la norma de la consulta es la piedra angular del Convenio (CEPAL, 2007; mi subrayado).

El 13 de septiembre de 2007 la Asamblea General aprobó la Declaración de las Naciones Unidas sobre los Derechos de los Pueblos Indígenas, que reconoce en el artículo 3 el derecho a su libre determinación, entendido como el derecho a determinar su condición política y perseguir libremente su desarrollo económico, social y cultural. (CEPAL, 2017).

En junio de 2016 la Asamblea General de la OEA y sus Estados miembros aprobaron la Declaración Americana sobre los Derechos de los Pueblos Indígenas, primer instrumento de este organismo en que se promueven y protegen los derechos de los Pueblos Indígenas. En junio de 2017 el Plan de Acción sobre la Declaración Americana sobre los Pueblos Indígenas (2017-2021) afirma que se compromete a los Estados miembros acerca de la creación de un mecanismo institucional que dé seguimiento a la implementación de la Declaración Americana, capaz de responder a problemas o asuntos serios en materia de tierras y recursos indígenas, protección ambiental, bienestar y autogobierno de los pueblos indígenas, incluidas la salud y la seguridad de las mujeres, las niñas y niños indígenas ( Ibíd.).

La política del SASI establece el principio de atención diferencial, resalta la importancia de "contemplar la especificidad cultural", fundamentos que afirman el derecho a promover la articulación de concepciones y cosmologías relativas a salud y enfermedad, organización social y sistemas de salud tradicional de los pueblos indígenas (dentro de la estructura organizacional biomédica). Al decir de un interlocutor Iny: "conciliar os dos lados".

El espacio terapéutico brasileño integra organizaciones públicas biomédicas, ONGS, etno - medicinas, grupos domésticos y terapias religiosas diversas; es habitado por composiciones y recomposiciones culturales que le otorgan características de mestizo e híbrido y así se define (SAILLANT, 2004).

Parte de lo registrado mediante narrativas y observaciones participantes efectuadas en los servicios de atención en Salud Indígena se describe a continuación de manera abreviada:

Dossiê Redes de Inter-aprendizaje: nuevas cartografías interculturales y algunas propuestas de transformación R. Articul.const.saber, 2020, v.5: e65684 
(1) Los profesionales de la salud se muestran afectados por ejercer su práctica en un territorio que surge cotidianamente conflictivo y fronterizo. Transitan en "dos mundos culturales" experimentando aspectos vinculados a la heterogeneidad simbólica que habita la identidad indigena de manera no complementaria y opuesta radicalmente a su praxis. Esto se sintetiza focalizando el poder y la eficacia en lo biomédico (PEREIRA, 2012).

(2) Cuando se mencionan referencias culturales indígenas relativas a la salud y enfermedad son focalizadas en la figura del Paje, la alimentación tradicional indígena u otras costumbres destacadas como "diferentes" son identificadas a modo de inconveniente, obstáculo y como prácticas homogéneas que envuelven a todas las etnias en general. Estos aspectos no son concebidos como formas de aprendizaje o enriquecimiento de conocimiento, no se observa una actitud que habilite a reflexionar sobre prácticas de pluralismo médico concretas.

(3) La praxis cotidiana que reúne significados terapéuticos, políticos e ideológicos diversos en relación a la administración y tratamiento de la salud genera tensiones jerárquicas. Esto se dirime disociando y dejando bien separadas: la zona del sistema biomédico omnipresente (con sus correspondientes enunciaciones, prácticas administrativas y disciplinares) y la zona de lo tradicional y cultural distinto. Encapsulando esta última con un claro bies etnocéntrico como posible generadora de mayores interferencias.

La Cultura surge como una frontera- esencia a ser esclarecida y superada. Se resalta la existencia de obstáculos culturales que entorpecen un desenvolvimiento más acertado y efectivo en la política de atención en salud indígena con soporte financiero del Estado. Así se explicarían las negligencias, enfrentamientos y contradicciones confinando y reificando $L a$ Cultura como sustancia inmanente (FASSIN, 2002). Las circunstancias históricas de los pueblos indígenas, la historicidad y desenvolvimiento paradójico de las políticas y economías de vida a las que están sujetos o de las que han sido objeto, no se tienen en cuenta (FASSIN 2002; VICTORA, 2011).

"Eles tem uma cultura diferente" es una de las frases más registradas durante la etnografía de parte de los agentes de salud no indígenas. Aspecto que lleva a cuestionarse si

Dossiê Redes de Inter-aprendizaje: nuevas cartografías interculturales y algunas propuestas de transformación R. Articul.const.saber, 2020, v.5: e65684 
resulta instrumental este estilo de política multicultural como válvula de escape en la tensión que se presenta con cierta frecuencia en los territorios donde convive la diferencia cultural (HALL, 2010).

Según Tzvetan Todorov la actitud de tomar los valores propios como universales no sería una característica europea sino de cualquier población:

Tenemos dificultades para vernos a nosotros mismos a través de los ojos del otro como distintas instancias de una misma humanidad y percatarnos que somos un lugar de reencuentro de todo lo que nos ha influenciado" (TODOROV, Entrevista PUCP, 2012).

El disfrute del derecho a la salud de los pueblos indígenas se relaciona con el ejercicio de otros derechos, como por ejemplo la preservación de sus territorios y la protección ambiental correspondiente. A su vez existen determinantes sociales y culturales que inciden en el pleno goce de tales derechos: la discriminación estructural, dificultades en aplicación del consentimiento previo libre e informado, la no participación en la adopción de decisiones; la violencia, la falta de consideración de referencias indígenas como sus sistemas de creencias y modos de organización social y toda circunstancia que oficie de limitación para concebir la continuidad cultural basada en la interculturalidad (CEPAL, 2007).

En el cotidiano de los servicios de salud indígena explorados, se identifica un multiculturalismo conservador con un marcado apremio en asimilar (integrar) la "diferencia indígena" a las formas burocráticas "do homem branco" y a la tradición biomédica hegemónica (HALL, 2010).

Un "homem branco", tori - trabajador de la salud que a su vez no constituye una entidad homogénea y tampoco representa o alcanza cierto ideal brasileño de fusão racial (SAILLANT, 2004). Ideal que surge como sustrato ideológico de las políticas étnicas y parecería ser colocado como una cúpula mágica y protectora encima de la identidad indígena, para que calce en las políticas de inclusión.

Siguiendo estas líneas de reflexión en la XI Conferencia Regional sobre la Mujer de América Latina y el Caribe, efectuada en la ciudad de Brasilia en julio del año 2010 se reconoció una vez más que la salud integral de las mujeres constituye un derecho fundamental que implica la interacción de factores sociales, culturales, biológicos y que la desigualdad de género forma parte de los determinantes sociales de la salud (CONSENSO BRASILIA, 2010).

Dossiê Redes de Inter-aprendizaje: nuevas cartografías interculturales y algunas propuestas de transformación R. Articul.const.saber, 2020, v.5: e65684 
Figura 2 - Aulas de palha dirigidas por Iraci esposa del cacique Raúl de la aldea Buridina.

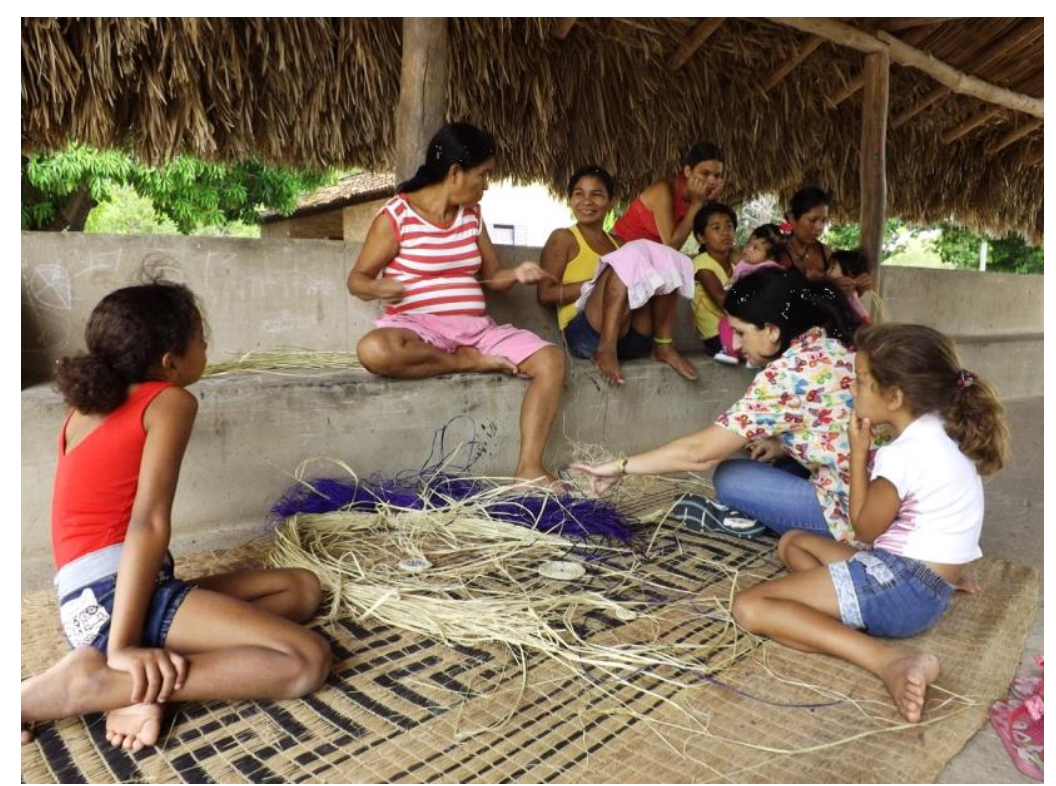

Fuente: Jacqueline Ledesma (Octubre 2013).

A su vez la Conferencia Global de Mujeres Indígenas realizada en Lima en 2013 reafirma que es preciso garantizar modelos de salud intercultural que respeten y apliquen sistemas simbólicos de organización y conocimientos tradicionales de salud de sus pueblos. (CONFERENCIA GLOBAL MUJERES INDIGENAS, 2013).

El multiculturalismo liberal y una perspectiva intercultural funcional se hace presente, en el cometido de integrar a los pueblos indígenas, otorgarles la posibilidad de ser ciudadanos y usuarios del SASI; siempre y cuando sus prácticas culturales (de salud u otras) queden fuera de un ámbito compartido socialmente, como se observa en el DSEI en el que se realizó la etnografía (WALSH, 2002; HALL, 2010).

Un multiculturalismo más pluralista se identifica en la redacción de las políticas en salud indígena y en el plano de ejecución que parece depender de la voluntad y actitud de ciertos equipos e individuos. El respaldo-reconocimiento de las diferencias y/o el descentramiento etnocéntrico es referido en algunos trayectos de trabajo a personas y equipos identificadas como: “indigenistas" o "mais envolvidos na causa indigena", que parecen poseer sensibilidades particulares en la hora de vincularse con los pobladores de las aldeas de Aruanã.

Siguiendo las reflexiones de Slavoj Zizek parecería que las configuraciones vinculares y comunicacionales que surgen en el confronto entre lo universal y lo particular, en el 
desarrollo de "estrategias de conformidad multicultural", se resuelven absorbiendo, incorporando o exagerando ciertos rasgos de aquello definido como: popular, étnico o diferente, vaciándolo de contenido y neutralizándolo. Reintegrándolo más depurado y funcional al plano de las relaciones hegemónicas de dominación y explotación (ZIZEK, 1998).

Las políticas multiculturales o interculturales que desenvuelven distintos países en América del Sur (Brasil, Bolivia, Ecuador, México) constituyen estrategias que intentan responder a perspectivas homogeneizantes en los campos de la salud y la educación; procuran propiciar el diálogo interétnico o se transforman en posturas funcionales a la desigualdad y la diferencia ( DIEZ, 2004).

En países que se postulan emblemáticamente, como "democracias de cosmovisiones diversas", las políticas concebidas con un enfoque intercultural receptivo al intercambio crítico y troca de saberes, en su desarrollo pueden transformarse como los diversos matices del multiculturalismo sustantivo, en programas integracionistas/asimiladores, objetivándose en el mero control/ contención de los conflictos y equilibrio social (WALSH, 2002).

Lo intercultural siempre ha existido el desafío es que acontezca en los territorios cotidianos que son conflictivos a manera de encuentro dialógico para establecer, puentes y mediaciones de comunicación reciprocas, capaces de superar estereotipos donde lo propio (mistura de lo cultural, nacional, étnico) tenga lugar y no se fagocite (WALSH, 2009; RIZZO, 2006; SAÉZ, 2005).

En el año 2015 Victoria Tauli-Corpuz Relatora Especial de la Organización de las Naciones Unidas sobre los Derechos de los Pueblos Indígenas, luego de realizar visitas a los países de la región para apreciar el seguimiento y cumplimiento de los estándares en derecho, expresa en uno de sus informes que se evidencian graves desigualdades en la atención en salud física y mental de la población indigena. (ONU; 2015; A/HRC/30/41).

A su vez llama la atención sobre los sistemas de salud afirmando que: "a menudo no tienen en cuenta el concepto indígena de salud y en consecuencia, crean barreras para el acceso de los pueblos indígenas" (p. 10; ONU, 2015, A/HRC/30/41).

La relatora destaca que los modos de integración de los sistemas de salud no son siempre claros para: el personal de salud, las comunidades y sus consultantes, los que diseñan las políticas y los funcionarios públicos. A lo cual se suma que las instalaciones en que se

Dossiê Redes de Inter-aprendizaje: nuevas cartografías interculturales y algunas propuestas de transformación R. Articul.const.saber, 2020, v.5: e65684 
desenvuelve la atención en salud, no se adaptan a preferencias culturales y necesidades específicas (ibíd.).

De acuerdo a lo que relatan los manuales consultados de la Comisión Económica para América Latina y el Caribe (CEPAL) específicamente en el publicado en el año 2017 cuyo título es significativo: "Los pueblos indígenas en América (Abya Yala) desafíos para la igualdad en la diversidad"; en la medida que se ha avanzado en el reconocimiento de los derechos individuales y colectivos de los pueblos indígenas, la concepción de salud intercultural se ha amplificado trascendiendo el intercambio de pluralidades en el territorio de los dispositivos de salud y el conocimiento y aceptación de la diversidad cultural (CEPAL; 2017).

Para el ejercicio pleno del derecho a la salud de los pueblos indígenas como colectivos diferenciados la praxis en salud intercultural, tendría que abarcar: el fomento de cambios en la organización y la oferta de los servicios de salud, acciones para potenciar la medicina tradicional indígena (incluyendo a las personas portadoras de conocimiento tradicional y símbolos), la investigación y resolución de otros factores estructurales de exclusión. Y plantearse estrategias para favorecer la coordinación horizontal entre ambos sistemas de salud (ibíd.).

El contexto etnográfico descripto, no es percibido por muchos de sus participantes como un ámbito de poder denso en él cual se intentan procesar las diferencias culturales; un "entre medio" donde algunos se encuentran y otros permanecen más excluidos, menos escuchados y/o separados. Espacio interesante en la hora de elaborar y pensar estrategias de representación cultural e identitaria; teniendo en cuenta que en sus dibujos los Iny recurren al poder de sus tradiciones, como capital simbólico eficaz, a ser re inscripto en las políticas de representación en salud y enfermedad indígena. (BHABHA, 1996).

Stuart Hall afirma que los escenarios multiculturales en los cuales se procura administrar identidades múltiples se encuentran en una permanente re-composición dinámica confrontativa, generando una tensión que provoca el pensamiento verdaderamente productivo y subraya la necesidad de vivir esas tensiones, "sin garantías" (HALL, 2010).

Es preciso pensar la interculturalidad como significante y concepción en construcción posible de ser interpelado/ evaluado en la praxis cotidiana por los sujetos que protagonizan y participan de las diversas economías y políticas de vida en las que son "beneficiarios".

Dossiê Redes de Inter-aprendizaje: nuevas cartografías interculturales y algunas propuestas de transformación R. Articul.const.saber, 2020, v.5: e65684 
Por otra parte resultaría interesante considerar los vínculos interculturales en salud indígena desde una perspectiva crítica, como propuestas, actitudes y procesos conflictivos que están siendo y expresan claramente asimetrías, violencias simbólicas y concretas. Que en su contrapunto producen: un quantum de conocimiento crítico y creativo, un plus de poder simbólico y epistemológico provocante que por veces queda oculto, es negado o no se le permite emerger con todo el potencial que posee.

\title{
2. Derechos Humanos tan lejos y tan cerca
}

\begin{abstract}
Entretanto, na pratica as ações de efetivação desses direitos ainda são muito fragmentadas. E uma luta árdua para os conquistados serem reconhecidos e seus direitos garantidos como povos específicos. Ainda se trata de uma legislação "abstrata" em relação a realidade vivenciada pelos povos indígenas brasileiros que vivem hoje nos vários estados do território nacional (DAMSKẼKWA XERENTE, p. 388, 2017).
\end{abstract}

Las políticas públicas en salud indígena promueven y aceptan la convivencia de diferentes culturas, como otras estrategias destinadas a gestionar el multiculturalismo que habita el Brasil, contienen preceptos éticos jurídicos universales, regionales y locales, para ofrecer un marco garantista de cumplimiento de lo reglamentado y a su vez fortalecer la cohesión social.

Las construcciones sobre derechos humanos (ddhh) caracterizadas por un intenso bies generalizador y una praxis distante de la realidad subjetiva de las comunidades indígenas; son interpeladas por los Iny y los trabajadores tori del DSEI Araguaia.

Podríamos afirmar que la concepción y representaciones de los derechos humanos son resultado de los imponderables de la convivencia humana, de múltiples aprendizajes, sufrimiento social y cultural. Los ddhh son inherentes a la persona humana, nacemos con derechos que han sido fruto de constantes transformaciones sociales y culturales; son universales, indivisibles e interdependientes (CORREA, 2017).

La protección de los derechos humanos se va construyendo jurídicamente a lo largo de la historia, el catálogo de derechos se amplia y alimenta de las diversas circunstancias por las que atraviesan y se ven afectadas personas, grupos y comunidades. Forman parte de esas eventualidades los procesos de discriminación, estigmatización y etnocentrismo. (ibíd.).

Dossiê Redes de Inter-aprendizaje: nuevas cartografías interculturales y algunas propuestas de transformación R. Articul.const.saber, 2020, v.5: e65684 
La discriminación alude a la violencia simbólica y física de la que son objeto personas y grupos por motivos: étnicos, de tradición cultural distintita, de género, sexualidades, religiosos, políticos, generación; situación de salud y enfermedad. El estigma refiere a actitudes de rechazo, desacreditación, falta de aceptación y temor hacia aquellas personas o grupos, en los que se colocan marcadores de diferencia variados (op. cit).

El fenómeno del etnocentrismo puede ser entendido como una reacción defensiva en situaciones en las que el otro se experimenta como amenaza a la identidad. El contacto con alteridades "catalogadas como diversas y distintas", en referencia a modelos esencialistas u homogéneos del Ser Humano, moviliza una mezcla de afectos y procesos racionales e intelectuales, ante las dificultades producidas en la interrelación de formas de ser y estar en el mundo, que se desean mantener con una frontera que paute la pertenencia a universos distantes y/o distintos (op.cit).

Los procesos vinculares señalados afectan, sino todos por lo menos un par de los presupuestos fundamentales de los derechos humanos: "Todos los seres humanos nacen libres e iguales en dignidad y derechos".

Existen individuos, grupos y colectivos que históricamente han sido y son objeto de tales procesos, para los mismos se han redactado derechos y protecciones más específicas (SALGUEIRO, 2008). Se identifican como grupos vulnerables; cuando es más acertado señalar que son grupos: subalternizados, vulnerabilizados, víctimas de procesos de exclusión, desigualdad social y exterminio.

Algunos de estos grupos en situación especial, serían: las mujeres, los niños, niñas y adolescentes, los pueblos indígenas, grupos afro descendientes, adultos mayores, personas con discapacidad, afectadas por sufrimiento mental, personas refugiadas, desplazadas y migrantes; personas con determinada preferencia sexual (LGTBI), personas privadas de libertad. (SALGUEIRO, 2008).

Es preciso tener en cuenta en las reflexiones y diseño de políticas sociales y culturales para "la diversidad" que la generación, y la condición de salud y enfermedad se articulan de distintas maneras con otros rasgos de identidad social y marcadores en un mismo sujeto social/sujeto de derecho. Como por ejemplo: la orientación sexual e identidad de género, la desigualdad y exclusión social, el conflicto con la ley, el origen étnico. Estos aspectos y contextos definen de diverso modo el alcance y acceso a las lógicas de aceptación, inclusión y/o mayor exclusión social (BRAZ; MELLO, 2011).

Dossiê Redes de Inter-aprendizaje: nuevas cartografías interculturales y algunas propuestas de transformación R. Articul.const.saber, 2020, v.5: e65684 
Los organismos internacionales e interamericanos redactan manuales y textos relativos al conocimiento e información "necesarios" para garantizar la protección de los ddhh políticos, sociales y culturales, se publican guías de la OMS (Organización Mundial de la Salud) UNESCO (Organización de las Naciones Unidas para la Educación, la Ciencia y la Cultura) UNICEF (Fondo de las Naciones Unidas para la Infancia) CIDH (Comisión Interamericana de Derechos Humanos). Las representaciones de estos organismos en cada país ofrecen conferencias, diversos tipos de cursos y sistemas de actualización, en materia de derechos humanos.

Una vez que las declaraciones, tratados, convenciones se declaran vigentes los Estados cuando los ratifican, promulgan y reúnen leyes que reconocen los derechos humanos, posteriormente tendrían que actualizar sus instituciones para efectivizar y garantizar el ejercicio de los mismos. El Estado Nación tendría que ser el principal promotor y defensor de los ddhh de los individuos, creando mecanismos de información, protección, prevención y denuncia.

Las extensas y densas construcciones sobre ddhh pueden ser analizadas para su comprensión desde el punto de vista histórico, sus fundamentos teóricos, de soporte conceptual y origen situacional. No se circunscriben a un ámbito puramente jurídico, las personas y grupos sociales han padecido de falta de información/protección y han tomado contacto con sus derechos en circunstancias graves- urgentes de afrenta a su integridad, a la de su familia o comunidad cultural a la que pertenecen. Es en los itinerarios de los servicios de salud o de otras instituciones donde transcurre la vida donde los preceptos a proteger no se cumplen (CORREA, 2017).

Esto remite a la dimensión pragmática/experiencial de los mismos y la necesidad de mecanismos, herramientas y diálogos interdisciplinarios que permitan las garantías necesarias para trascender el plano de lo prescriptivo a la vivencia y práctica cotidiana (URIBE, 2011).Las personas además de comprender el significado real y simbólico de ser un sujeto de derechos titular, necesitan tener las condiciones de existencia necesarias para disfrutar de los mismos.

De lo contrario la perspectiva de derechos humanos se vacía de significado y pierde su potencial como herramienta de lucha contra la desigualdad, exclusión y la injusticia.

Siguiendo estas reflexiones vinculado al derecho a la salud y la educación de los pueblos indígenas se encuentra el derecho a la alimentación que habría de incluir la dimensión

Dossiê Redes de Inter-aprendizaje: nuevas cartografías interculturales y algunas propuestas de transformación R. Articul.const.saber, 2020, v.5: e65684 
de identidad étnica. Uno de los profesores de la Escola Maurehi que vivía en la aldea BdèBrè cuyo nombre es Gedeón plasmo en un par de dibujos sus concepciones acerca de la salud y enfermedad relacionándolas con la alimentación tradicional y la tori.

Figura 3 - Diseño (1)

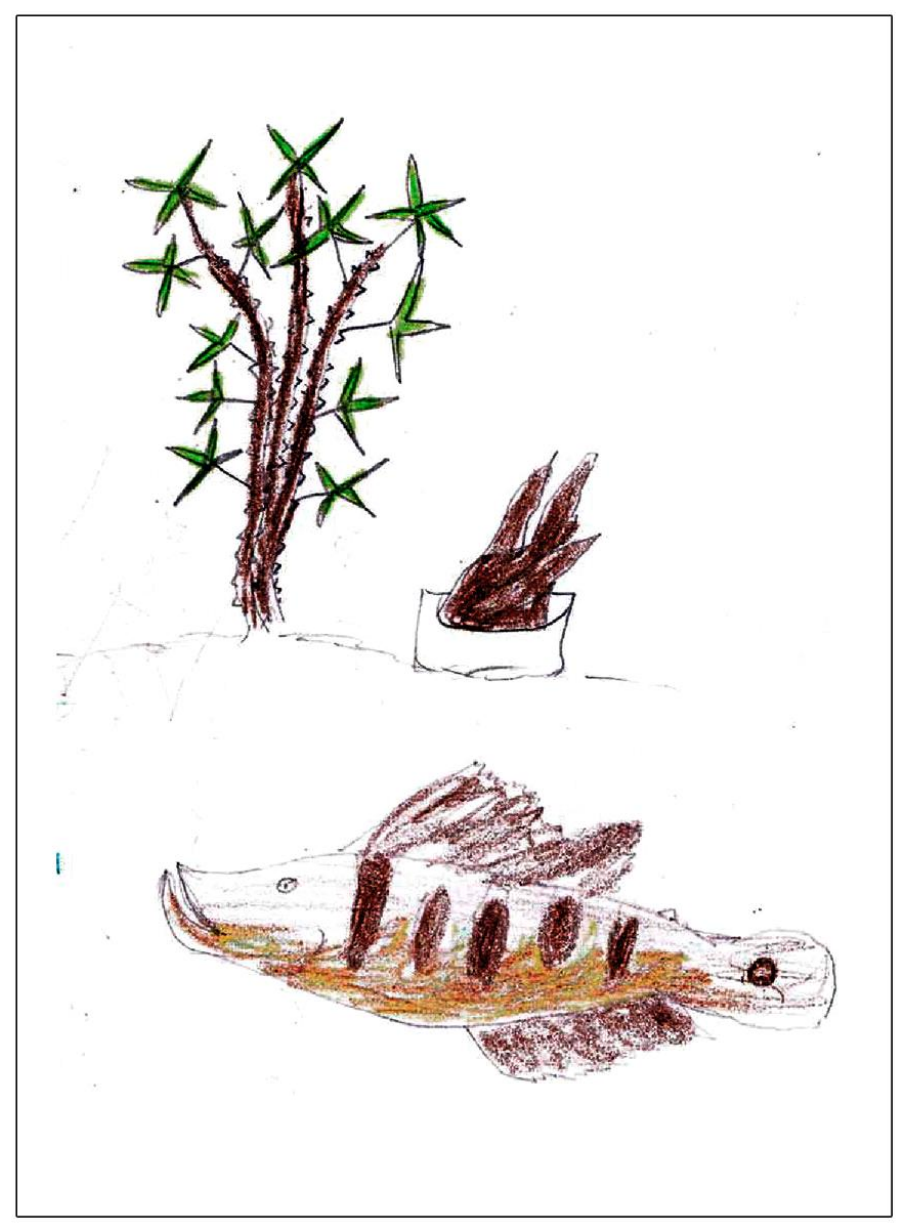

Fuente: Gedeón. Profesor de Escola Maurehi, padre habitante de Aldea Bdè-Brè (Octubre 2013).

Su primer dibujo expresa la salud, tal como él describió son alimentos de la naturaleza y "alimentación tradicional Iny": una planta de mandioca en la tierra y luego la misma en un recipiente lista para quitar su cáscara, rallarla y cocinarla. El pez es un pirarucú, que tendría que ser asado al fuego de forma natural para su consumo. "Esta é a saúde, não veneno ou tóxico, agora tudo alimento tem veneno. Iny e magro não tem porque ter problemas de saúde tem que fazer arroz na panela de palha e procurar peixe asado uma vez por dia". (CORREA, 2014).

Dossiê Redes de Inter-aprendizaje: nuevas cartografías interculturales y algunas propuestas de transformación R. Articul.const.saber, 2020, v.5: e65684 
Cuando le consulte acerca de que significaba para él la enfermedad me expuso su definición narrada en otro dibujo: “TV. óleo refrigerante, isso é doença. Crianças não quer fazer nada com a TV. Eles ficam pensando em coisas da TV, é ruim para a saúde”. "Refrigerante muito açucarado, doce e óleos e gorduras não são boas para a saúde, tudo o que nos prejudica a saúde é o que nós comemos" (ibíd.).

Figura 4 - Diseño (2)

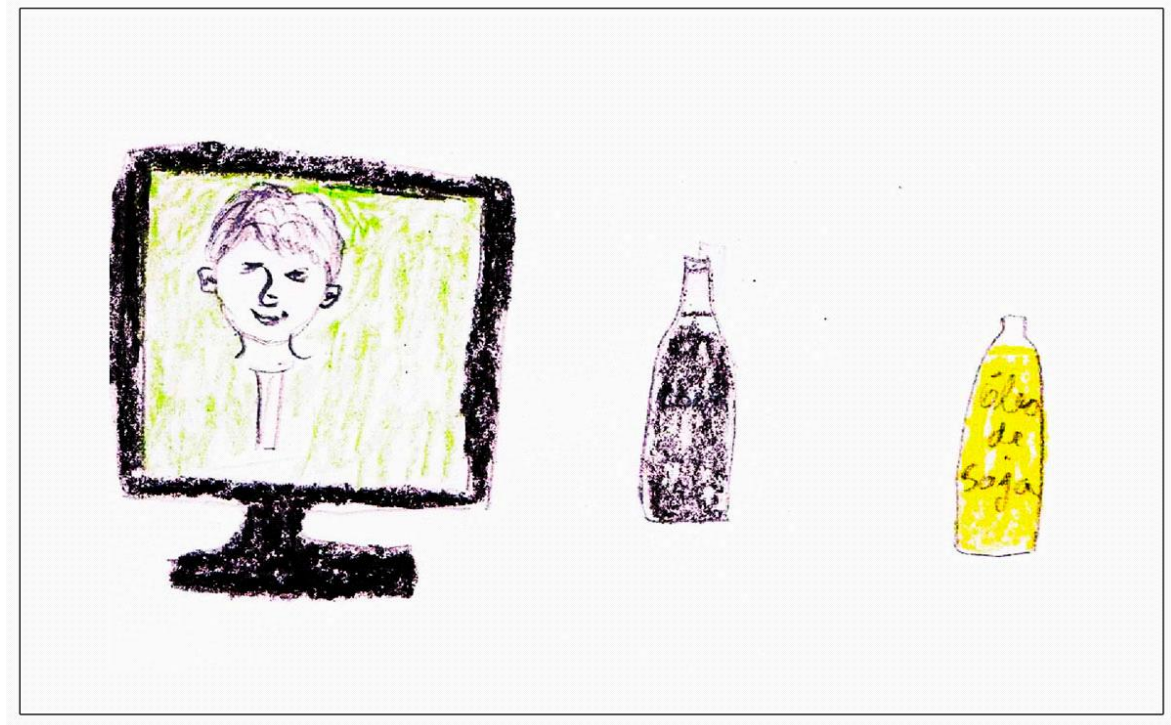

Fuente: Gedeón. Profesor de Escola Maurehi, padre habitante de Aldea Bdè-Brè, (octubre 2013).

Las narrativas visuales producidas por el joven profesor reflejan una fuente de preocupación que es planteada de forma recurrente por los pobladores Iny de Aruanã, algunos agentes de salud del CASAI y de los servicios de salud de Aruanã. Las dificultades con la alimentación y su relación con los tratamientos de enfermedades que según unos y otros interlocutores son producto del contacto interétnico, sumado a los problemas que poseen algunas familias numerosas, para conseguir alimentos. Y realizar una dieta más sana que es asociada a los alimentos naturales y tradicionales de la dieta Iny (ibídem.).

Tal como se afirmó en la etnografía realizada en Aruanã las concepciones de salud y enfermedad del pueblo Iny surgen y son interpretadas, como aspectos del cotidiano inmersos en una trama de relaciones amplia que reúne: relaciones políticas, sociales, culturales, económicas, interculturales, que las delimitan y definen (GARCÍA, 2009). 
La doctora en historia y antropología social de la Universidad de Brasilia Poliene Soares dos Santos Bicalho, efectúa un delicado trabajo de investigación en su tesis de doctorado, Protagonismo Indigena no Brasil: Movimento, Cidadania e Direitos (1970-2009). Parte de su investigación consistió en entrevistar en el año 2008 a mujeres y hombres indígenas que ocupaban cargos de dirección, coordinación y asesoría en diversas organizaciones de lucha y representación de los pueblos indígenas.

Una de las preguntas formulada por la pesquisadora consistió en que sus interlocutores describieran las demandas de parte del Movimiento Indígena en la década de 1970/1980 y lo que se solicitaba y esperaba en el actual siglo XXI.

Varios entrevistados coinciden en destacar que en los inicios las luchas estuvieron marcadas por una urgente demanda de visibilidad, para tener derecho a emitir su voz y ser escuchados, no ser más discriminados, detener las constantes maniobras de expulsión e invasión de sus tierras y que las mismas se demarcaran. No es vano recordar que los indígenas eran tutelados y considerados incapaces; figura del derecho que implica el no reconocimiento de la propia voluntad, el carecer de autonomía jurídica y entre otros detalles humanos no poder ser titular de derechos y obligaciones (BICALHO, 2010).

Por otra parte sostienen que el imperativo en el siglo presente es garantizar la participación en el diseño y ejecución de las políticas públicas y ser los protagonistas reales en las gestiones que tienen lugar dentro de sus tierras. Aspectos que han de ser efectivizados de hecho y no depender de la voluntad de la administración política gubernamental de turno (ibíd.).

Resulta paradójico pensar que ciudadanos habitantes de un país, sujetos de derecho que no son seres abstractos tengan que efectuar reclamos desde el respeto de su identidad distintiva y se vean envueltos en una especie de concesión de favores especiales. Cuando se trata en el devenir histórico de los pueblos indígenas, de una lucha ancestral en contra de la colonización violenta y la concreción de agencialidad social y cultural que ha sido muy postergada (KENRICK, 2009).

Otro detalle importante que manifestaron trabajadores de la salud del pueblo Iny, referentes comunitarios de las aldeas de Aruanã y las personas entrevistadas por la profesora Poliene Soares, es que si bien se ha avanzado, los derechos no abarcan o responden a la realidad heterogénea de los pueblos indígenas brasileños. A lo que se suma como critica que el Estado Nación brasileño no adecua sus estructuras políticas ya que se resiste a modificar de

Dossiê Redes de Inter-aprendizaje: nuevas cartografías interculturales y algunas propuestas de transformación R. Articul.const.saber, 2020, v.5: e65684 
manera sustancial sus instituciones para que exista una real, efectiva concreción y protección de los derechos indígenas (BICALHO; 2010).

\section{Consideraciones Finales}

La intención de este escrito fue procurar una aproximación en el análisis de estilos de administración multicultural y comunicaciones interculturales observadas en la etnografía sobre políticas públicas en salud indigena realizada con el pueblo Iny de la ciudad de Aruanã y los trabajadores de los espacios de atención en salud del DSEI Araguaia.

Lo que se plantea describe en cierto modo un campo de relaciones complejas en el cual la asistencia en salud se desenvuelve entre medio de confrontaciones que surgen por momentos como imposibles de resolver de una manera que no sea otra que separar y controlar más una de las múltiples alteridades étnicas que habita en Brasil.

La política pública en salud indigena está diseñada y llevada a cabo principalmente y en su mayoría por la perspectiva administrativa, biomédica y burocrática tori, con una minoritaria representación indigena.

Como ya se señaló "lo diferente" no es percibido o incorporado desde su conflictividad productiva, como alimento social-cultural enriquecedor y poseedor de desconocidas enseñanzas; se continúa disociando como elemento/ ajenidad incomoda e impura. Aspecto que lleva a preguntarse qué es lo que está dispuesto a aprehender el mundo tori de los Iny y de los pueblos indígenas del Brasil, en concepciones de salud y enfermedad o en referencia a otras áreas de interés políticas y culturales.

En octubre de 2013 al ir finalizando el trabajo de campo un par de trabajadores de la salud Iny me planteaban su preocupación respecto a que la política de salud del SASI, pasara a manos de la prefeitura (municipalidad). Lo cual ponía en riesgo el derecho a una política Étnica Estatal Federal producto de años de lucha y la atención en salud propiamente dicha ya que según mis interlocutores, el subsistema de Salud Indígena en su complejidad no puede sostenerse por las prefeituras.

Al momento de efectuar este artículo (2019) el gobierno brasileño proponía cerrar el subsistema de Salud Indígena amparado por la Secretaría Especial de Salud Indígena (SESAI) y eliminar la Secretaria para municipalizar los servicios y la asistencia médica indígena. " $E l$ subsistema de salud indígena es nuestra conquista y tiene que ver con nuestra lucha. No

Dossiê Redes de Inter-aprendizaje: nuevas cartografías interculturales y algunas propuestas de transformación R. Articul.const.saber, 2020, v.5: e65684 
pertenece a un partido o a cierta política, ya que lo construimos nosotros". (Elói Jacinto, líder indígena guaraní, 26/03/2019; telesurtv.net.).

¿Porque no evaluar críticamente el desenvolvimiento de la política en salud existente de acuerdo al contexto de cada DSEI desde la perspectiva de los representantes indígenas y las familias que se atienden y proponerse avances? ¿Por qué debilitar la Salud Indigena y los logros del Movimiento Indígena Brasileño? Interrogantes que abarcan intereses diversos y requieren múltiples profundizaciones.

En la etnografía efectuada, algunas familias Iny expusieron preocupaciones y circunstancias cotidianas posibles de ser referidas a un aspecto de la salud integral, que la epistemología occidental conceptualiza como: salud emocional, salud mental, salud afectiva. En parte se describieron situaciones de consumo problemático de sustancias legales e ilegales, a su vez la violencia, el sufrimiento y la inestabilidad que genera convivir con el riesgo permanente de perder sus tierras o las políticas públicas educativas y de salud conquistadas. Estos hechos manifiestan y denuncian una vez más el contexto situacional de fragilidad y vulneración en el que experimentan sus vidas los pueblos indígenas en el presente, en el cual el derecho fundamental a la vida se encuentra con claros límites y en riesgo.

Tal como mencionan las maestras de cerámica y palha, las actividades de elaboración y aprendizaje de artesanato, junto a la tarea de la Escola Maurehi configuran un soporte afectivo -social y cultural importante vinculado al resguardo y trasmisión de la cosmología y tradición Iny. Las narrativas visuales que registran su tarea comunican la idea de salud y terapéutica particular como prácticas tradicionales cuya eficacia simbólica influye en la salud y bienestar del pueblo.

Sería interesante continuar investigando con los habitantes de las aldeas estas consideraciones desde una perspectiva intercultural crítica, de género y generaciones, que incorpore la medicina tradicional y el sistema de significados del pueblo Iny del rio Araguaia.

El campo de problemas de los Derechos Humanos es denso, dilemático y complejo, abarca nociones de sujeto individual, sujeto de derecho y derechos de grupos y colectivos. En el contexto de los pueblos indígenas navega entre las particularidades de la identidad étnica, la pluralidad de pueblos y la versión occidental universal de hombre adulto céntrico- blanco.

Las convenciones y declaraciones pueden extrañarnos percibiéndolas como formales construcciones etnocéntricas, por otra parte fueron y son conjuntos de preceptos que actúan 
como marco de protección y plataforma de demanda frente a la desigualdad y la violencia política y social.

En esta línea de reflexión voy a citar palabras de Marcos Terena líder indígena del Povo Xané (fonéticamente Uxtexané, que significa “Eu sou Eu”) figura emblemática del Movimento Indígena do Brasil en la entrevista que le efectúa Poliene Soares:

\begin{abstract}
Qual o direito do índio? Nós precisamos regulamentar esses novos critério (sic) de direito. E nós, uma coisa interessante, apesar de não termos formulado isso no Brasil, na arena internacional, nós avançamos muito nos Direitos Humanos. Uma Declaração da ONU que, apesar de não ser advogado, participei, durante os últimos vinte anos. Agora, que assinou a carta, fomos nós? Não! Foi o Governo Brasileiro. Por que na ONU funciona assim, somos os convidados, tal, participantes, temo (sic) voz, mas o voto é do Governo Brasileiro; por que a ONU é formada pelos Estados. (Terena Marcos en: BICALHO; 2010, p. 406.)
\end{abstract}

¿Cómo se vinculan las personas beneficiarias de las políticas públicas de acción afirmativa con las construcciones discursivas de ddhh en su vida cotidiana? ¿Existe una necesidad de información/aprendizaje? ¿Quién confiere la autorización y potestad para realizar planes educativos al respecto y en que lenguaje se efectúan?

De manera recurrente en los múltiples materiales publicados por organismos internacionales e interamericanos surge la falta de formación de recursos humanos en materia de derechos. Lo que lleva a plantearse más interrogantes. ¿Cómo se introducen las perspectivas de ddhh en los cambios y reestructuras que han afectado y afectan la configuración organizativa de todas las instituciones que participan del sistema de atención en salud indígena?

Los trabajadores de los distintos DSEI como aprehenden e integran el enfoque de protección y garantías que incluye distintas dimensiones y especificidades en su tarea de atender procesos de salud y enfermedad ¿Los ddhh son un instrumento técnico? ¿Cómo se vinculan con los presupuestos epistemológicos y éticos de las disciplinas que intervienen en los itinerarios de atención en salud? y ¿con la praxis dialéctica intercultural?

¿Qué particularidades presenta el proceso de internalización y efectivización del conjunto de estándares? ¿Será que tienen una estructura interpretativa previamente asignada? ¿Porque algunos técnicos en derecho expresan con insistencia que es necesaria una toma de conciencia respecto a tales preceptos y un cambio de cabeza? ¿Existe lugar en estos razonamientos para tener en cuenta las complejidades que acontecen en los trayectos de comunicación y relación interinstitucional e intersectorial?

Dossiê Redes de Inter-aprendizaje: nuevas cartografías interculturales y algunas propuestas de transformación R. Articul.const.saber, 2020, v.5: e65684 
¿Es posible pensar en los modos de apropiación subjetiva por medio de los cuales, los derechos humanos se resignifican en un cotidiano donde coexisten personas, grupos, habitus y culturas institucionales que les concederán o no poder y eficacia simbólica en diverso grado y matices?

\section{Referências Bibliográficas}

BANIWA, Gersem dos Santos Luciano. O Índio Brasileiro: o que você precisa saber sobre os povos indígenas no Brasil de hoje. Brasília: Ministério da Educação, Secretaria de Educação Continuada, Alfabetização e Diversidade; LACED/Museu Nacional, 2006.

BARROS, Norami M.; CAIXETA, Leonardo F. Prevalência de Transtornos Cognitivos entre Idosos, Indígenas da Etnia Karajá. 2010-2011. Relatório Final (PIVIC). Universidade Federal de Goiás, Instituto de Patologia Tropical e Saúde Pública. Goiânia, 2011.

BHABHA, Homi K. El Lugar de la Cultura. Ediciones Manantial. Bs. As. 1996.

Comisión Económica para América Latina y el Caribe (CEPAL). Naciones Unidas. Panorama social de América Latina. 2007.

https://repositorio.cepal.org/bitstream/handle/11362/1227/S0700764_es.pdf?sequence=.

Comisión Económica para América Latina y el Caribe (CEPAL) Los Pueblos Indigenas en América Latina. Avances en el último decenio y retos pendientes para la garantía de sus derechos. 2014. https://www.cepal.org/es/publicaciones/37050-pueblos-indigenas-americalatina-avances-ultimo-decenio-retos-pendientes-la.

Conferencia Regional sobre la Mujer de América Latina y el Caribe. 16 de julio de 2010. Consenso de Brasilia. https://www.cepal.org/mujer/noticias/paginas/5/40235/ConsensoBrasilia_ESP.pdf.

CORREA, Isabel J. Os Itinerários Terapêuticos do Povo Iny-Karajá das Aldeias Buridina e Bdè-Brè de Aruanã (GO) Dissertação apresentada à Faculdade de Ciências Sociais da Universidade Federal de Goiás (UFG) para obtenção do título de Mestre em Antropologia Social. Brasil 2014.

Artículo inédito para cargo de Asistente del Área Derechos Humanos - Unidad Académica del Servicio Central de Extensión y Actividades en el Medio - Universidad de la República del Uruguay. Montevideo, Uruguay 2016.

Pesquisa y asesoría en salud mental/discapacidad/salud integral/conflicto con la ley y derechos humanos en infancias y adolescencias. Institución Nacional de Derechos Humanos y Defensoría del Pueblo/Mecanismo Nacional de Prevención a la Tortura (INDDHH/MNP) UNICEF (Fondo de las Naciones Unidas para la Infancia) Montevideo, Uruguay 2017.

Dossiê Redes de Inter-aprendizaje: nuevas cartografías interculturales y algunas propuestas de transformación R. Articul.const.saber, 2020, v.5: e65684 
Declaración de Lima de la Conferencia Global de Mujeres Indigenas, octubre 2013. ¡Mujeres Indígenas Hacia la Visibilidad e Inclusión! https://www.forestpeoples.org/es/topics/lascuestiones-de- $\quad$ genero/news/2013/11/declaracion-de-lima-de-la-conferencia-global-demujeres.

Del Popolo Fabiana (ed.), Los pueblos indígenas en América (Abya Yala): desafíos para la igualdad en la diversidad, Libros de la CEPAL, $\mathrm{N}^{\circ} 151$ (LC/PUB.2017/26), Santiago, Comisión Económica para América Latina y el Caribe (CEPAL), 2017.

DIEZ, María Laura. Reflexiones en torno a la interculturalidad. Cuadernos de Antropología Social No 19, pp. 191-213, 2004. UBA. Disponible en: www.scielo.org.ar.

Dos Santos Bicalho Poliene. Protagonismo Indígena no Brasil: Movimento, Cidadania e Direitos (1970-2009). Tese apresentada ao Programa de Pós-Graduação do Departamento de História como parte dos requisitos para a obtenção do Título de Doutora em História. Área de Concentração: História Social. Universidade de Brasília, 2010.

ERCIVALDO, Damskẽkwa Xerente. Os Direitos Humanos e a Diversidade Cultural a Partir da Experiência de um Acadêmico Indigena AKWÊ/ XERENTE, no Mestrado de Direitos Humanos da UFG. En: Educação indígena e interculturalidade: um debate epistemológico e político, Mariano Baez Landa; Alexandre Ferraz Herbetta (Org.). Goiânia: Editora da Imprensa Universitária, (PPGDH/ PPGAS/ UFG) 2017 Brasil. https://www.cegraf.ufg.br/up/688/o/ebook_educacao_indigena.pdf.

FASSIN, Didier. Exposición del Dr. DIDIER FASSIN (EHESS, Paris) en el Seminario: Sociedad y Cultura II. Facultad de Humanidades. UDELAR. Montevideo. 24 de junio 2002.

Fin a 17 años de espera para los pueblos indígenas. OEA aprobó la Declaración americana sobre los Derechos de los Pueblos Indígenas. En las Américas habitan 50 millones de personas que se autodefinen indígenas. https://www.oas.org/es/centro_noticias/comunicado_prensa.asp?sCodigo=C-075/16.

FUNASA, Lei Arouca. 10 anos de Saúde Indígena. Brasília: FUNASA, 2009.

FUNASA. Política Nacional de Atenção à Saúde e Povos Indígenas. Funasa (Fundação Nacional da Saúde) MS (Ministério Nacional da Saúde) Brasília, Março 2002.

GARNELO, Luiza; PONTES, Ana Lúcia. (Orgs.). Saúde Indígena: Uma Introdução ao tema. Brasília-DF: MEC/UNESCO, 2012.

HALL, Stuart. Sin garantías: Trayectorias y problemáticas en estudios culturales. Primera edición Agosto 2010 Envión editores. Popayán, Colombia.

Informe de la Relatora Especial sobre los derechos de los pueblos indígenas, Victoria Tauli $\begin{array}{lllllll}\text { Corpuz. } & \mathrm{A} / \mathrm{HRC} / 30 / 41 & 6 & \mathrm{de} & \text { agosto de } & \end{array}$ http://unsr.vtaulicorpuz.org/site/images/docs/annual/2015-annual-hrc-a-hrc-30-41-sp.pdf. 
KENRICK, Justin. The Paradox of Indigenous Peoples Rights en: World Anthropologies Network (WAN) Red de Antropologías del Mundo (RAM) electronic journal n. 4. Enero 2009. https://www.ram-wan.net/e-journal/.

LANGDON, Esther. Problematizando os Projetos de Medicina Tradicional Indígena. In: Medicina Tradicional Indígena en Contextos. Anais da I Reunião de Monitoramento. FUNASA. Brasília-DF, 2007.

MELlO, Luiz; Camilo Albuquerque de Braz. Políticas de saúde para lésbicas, gays, bissexuais, travestis e transexuais no Brasil: em busca de universalidade, integralidade e equidade. Revista Latino-americana / n. 9 - $\quad$ dec. 2011. http://www.scielo.br/scielo.php?script=sci_arttext\&pid=S1984-64872011000400002.

PEREIRA, Pedro Paulo Gomes. Limites, Traduções e Afetos: Profissionais de Saúde em Contextos Indígenas. In: Mana, N. 18 v. 3, p. 511-538. 2012. Disponível em: http://www.scielo.br/scielo.php?pid=S0104-93132012000300004\&script=sci_arttext.

RIZZO, Marta; SAEZ, Vivian. Cultura y comunicación intercultural. Aproximaciones conceptuales. Revista da Associação Nacional dos Programas de Pós-Graduação em Comunicação. Núm. vol. Agosto 2006. Disponible en: www.compos.org.br.

SAÉZ, Fernando T. En torno a la interculturalidad: reflexiones sobre cultura y comunicación para la didáctica de la lengua. Publicado en Porta Linguarum, $\mathrm{n}^{\circ}$ 4, 2005. Disponible en: www.ugr.es.

SAILLANT, Francine. Saber e itinerários de cuidados na Amazônia brasileira: o doméstico na encruzilhada de espaço terapêutico. In: LEIBING Anette (org.) Tecnologias do Corpo. Uma Antropologia das medicinas no Brasil. Rio de Janeiro. Edit. NAU. 2004.

SALGUEIRO M. Elena. Nociones Básicas en Derechos Humanos. Ministerio de Educación y Cultura. Montevideo, 2008.

Terena Marcos. Marcos Terena conta sobre os direitos dos povos indígenas a partir da narrativa de histórias ouvidas e vividas. Publicado em: 28/10/2019 - 22:30. Mekukradjá Círculo de Saberes. https://www.itaucultural.org.br/marcosterena-mekukradja.

TODOROV, Tzvetan. Conferencia en la Pontificia Universidad Católica de Perú (PUCP) 2012. Disponible en: www.youtube.com/watch?v=vvCilDszOJk

TORRES et. al., A Vida na Aldeia Versus a Vida na Cidade: O que pensam os indígenas da casa de saúde do índio Goiânia. Revista Estudos, v. 35, n. 11/12, p.1195-1210, nov./dez. PUC- Goiânia. 2008. Disponível em: http://seer.ucg.br/index. php/estudos/index.

URIBE, Enrique. Una aproximación epistemológica a los derechos humanos desde la dimensión vivencial pragmática. En: Boletín Mexicano de Derecho Comparado, nueva serie,

Dossiê Redes de Inter-aprendizaje: nuevas cartografías interculturales y algunas propuestas de transformación R. Articul.const.saber, 2020, v.5: e65684 
año XLIV, n. 132, septiembre-diciembre de 2011, p. 1233-1257 D.R. 2011. UNAM, Instituto de Investigaciones Jurídicas.

VERANI, Cibele B. A Política de Saúde do Índio e a Organização dos Serviços no Brasil. Boletim do MPG: Série Antropologia, v. 15, n. 2, p. 171-192. Dez. 1999.

VICTORA, Ceres. Sofrimento social e a corporificação do mundo: contribuições a partir da Antropologia. In: RECIIS - R. Eletr. de Com. Inf. Inova. Saúde. Rio de Janeiro, v.5, n.4, p. 313, Dezembro, 2011. Retirada do site: www.reciis.icict.fiocruz.br; em Dezembro de 2012.

WALSH, Catherine. Interculturalidad crítica y educación intercultural. En: ampliación de la ponencia presentada en el Seminario "Interculturalidad y Educación Intercultural", organizado por el Instituto Internacional de Integración del Convenio Andrés Bello, La Paz, 9-11 de marzo de 2009. Disponible en: https://aulaintercultural.org/2010/12/14/interculturalidad-critica-y-educacion-intercultural/.

WALSH, Catherine. (De) Construir la interculturalidad. Consideraciones críticas desde la política, la colonialidad y los movimientos indígenas y negros en el Ecuador. En: Interculturalidad y Política, Norma Fuller (ed.). Lima, Red de Apoyo de las Ciencias Sociales, 2002. Disponible en: www.uasb.edu.ec.

ZIZEK, Slavoj. Multiculturalismo o la lógica cultural del capitalismo multinacional. En: Estudios Culturales. Reflexiones sobre el multiculturalismo. Edit. Paidós. Bs. As. 1998.

Submetido em 16 de setembro de 2020.

Aceito em 23 de outubro de 2020.

Publicado em 27 de outubro de 2020. 